\title{
Numerical Simulation of Millimeter Wave Scattering by Foam Covered Flat Sea-surface modelled as Sequences of Thin Phase Scattering Screens
}

\author{
Ayibapreye K. Benjamin*, Collins E. Ouserigha**, Aguiyi Nduka Watson ${ }^{* * *}$ \\ ${ }^{*}$ Department of Electrical/Electronic Engineering, Niger Delta University \\ ** Department of Physics, Niger Delta University \\ ** Department of Electrical/Electronic Engineering, Federal University Otueke
}

DOI: 10.29322/IJSRP.11.10.2021.p11852

http://dx.doi.org/10.29322/IJSRP.11.10.2021.p11852

\begin{abstract}
This paper illustrates numerical results of millimeter wave scattering, based on a model of flat sea-surface covered by foams. This model considers the structure of foam as coated spherical particles that are randomly distributed within a cubic domain. The cubic domain is divided into five (5) two dimensional $2 D$ slices of foam layers. Electromagnetic wave with unit amplitude was propagated through each slice of sea-foam layer for accurate prediction of millimeter wave scattering due to the interaction between the EM wave and randomly distributed air-bubbles. The Finite difference method solution of the parabolic equation method was used in investigating diffused reflections from the foam-covered sea-surface. Results obtained using this method was compared with other experimental results which appear to show a good agreement.
\end{abstract}

Index Terms- Millimeter wave, Scattering, Foam covered sea surface, thin phase scattering screens.

\section{INTRODUCTION}

$\mathrm{T}$ he parabolic equation method is a one-way propagation model which is derived from the Helmholtz wave equation. By decoupling of Maxwell's equations, the Helmholtz equation is obtained [1,2]. Tappert was first to suggest the split-step Fourier solution for solving PE models [2]. This method was historically proposed initially by Tappert [3,4] in the early 1970s to solve the non-linear Korteweg-de Vries (Kdv) wave equation with constant coefficients. Hardin and Tappert presented a further application of the split-step Fourier transform (SSFT) solution to non-linear and variable coefficient wave equations [5]. Tappert [3] illustrated that the SSFT method is accurate and unconditionally stable for solving parabolic wave equation with variable coefficient. The split-step algorithm has been widely deployed to solve the SPE ever since it was developed by Hardin and Tappert [5] in the early 1970s. The technique is computationally efficient for long-range, narrow-angle propagation problems with negligible bottom interactions [2].

Reflection and refraction effects of millimeter wave $(\mathrm{mmW})$ interactions with densely packed particles (air-bubbles) are physical oceanographic and marine geological features [2]. In many scenarios, the three dimensional $(3 D)$ models are adequate for accurate prediction of electromagnetic wave (EM) field propagation through random media. Tolstoy [6] reported a variety of $3 D$ modelling techniques for describing EM wave propagation through random media over the past decades. In this article, we adopt the parabolic equation (PE) method, which was introduced by Tappert in the early 1970s for two dimensional (2D) one-way EM wave propagation problems. The PE Method is very effective and efficient in modelling long- range EM wave propagation in the ocean. However, propagation is mainly wide-angled with many bottom interacting paths for short-range, deep-water and shallow water problems.

The principal advantages of the various parabolic wave equations derived below is that it constitutes an initial value problem in range and hence can be solved by a range marching numerical technique, given a source field distribution over depth at the initial range.

Over the years, several different solution techniques have been implemented in computer codes [7], but only the split-step Fourier technique and various finite-difference/finite-element techniques have gained widespread use in the underwater acoustic community.

Before going into details on the numerical solution schemes, let us briefly point out some advantages and disadvantages of these two main solution techniques [2].

This requires the use of wide-angle PEs, which can be solved only by finite difference or finite elements. Moreover, the strong speed and density contrasts encountered at the water-bottom interface adversely affect the computational efficiency of the split-step technique, which in cases of strong bottom interaction requires an excessively fine computational grid $(\Delta x, \Delta z)$. Hence, the advantage of higher computational efficiency of the split-step technique is entirely lost in situations with strong bottom interactions [8]. 
Finite-difference and Finite-element solutions are applicable to PE for arbitrary large angles. The main drawback of these schemes is that, for long range the split-step solution is more efficient and also for narrow angle with minimal or no bottom interaction. The splitstep solution remains the most adopted technique for performance prediction as it is more suitable to solving many practical oceanic surface problems [9].

Conversely, the finite-difference and finite-element schemes have widespread application for wide-angle and bottom interacting boundaries. It is prominent for providing higher accuracy in these domains.

The most recent development in terms of efficient PE solution schemes is a split-step Pade' approximations derived by Collins [10]. He uses higher order Pade' approximations not the square root operators. The result is a considerable efficiency gain through the use of higher range step. Thus, the scheme is claimed to be more than an order of magnitude faster than standard FD/FE solution techniques. This could create a unified PE solution approach where the accurate high angle PEs can be solved with the efficiency of the classical split-step Fourier scheme [2].

\section{EVALUATION OF EFFECTIVE DIELECTRIC CONSTANT OF SEA-FOAM}

Figures A-E in [11] comprise of 3D (three dimensions) slices of randomly packed spheres in a unit cube. These slices were later translated to 2D slices of randomly packed circles. The conversion of 3D randomly packed spheres to 2D packed circle was achieved by calculating the radii of each individual circle using the concept of intersection of a sphere and a plane. The $2 \mathrm{D}$ slices were discretized with grid sizes $\Delta x$ and $\Delta y$ which leads to intersection of the circles bounded in a unit square with some grid points. The grid sizes were sampled such that the edges of the circles circumference which intersects with grid points farther from the inner grids bounded by the circles are negligible.

Estimate of the effective dielectric constant of sea foams were made by modelling the randomly packed bubbles as concentric circles in 2-D where the outer circle is a mixture of air and seawater while the inner circle contains about (80 - 95) \% air, with these estimates we were able to calculate the area of the annulus (ring) as the radii of the outer circles are known [11].

The dielectric constant of seawater at fixed salinity $34 \mathrm{psu}$ and sea surface temperature $20^{\circ} \mathrm{C}$. The dielectric constant of air is taken as $1.00005+0.0000 i$. The area of the circles in each slice was calculated using the total number of grid points. The effective dielectric constants of sea foams at frequencies $10.7 \mathrm{GHz}$ and $37.0 \mathrm{GHz}$ were calculated for 5 slices of randomly packed air-bubbles coated with thin layer of seawater [2].

$\begin{array}{ccc}\text { FREQUENCY } & 10.7 \mathrm{GHz} & 37 \mathrm{GHz} \\ \text { Slice 1 } & 1.0948-0.1251 \mathrm{i} & 1.0006-0.0332 \mathrm{i} \\ \text { Slice 2 } & 1.1248-0.1507 \mathrm{i} & 1.0108-0.0239 \mathrm{i} \\ \text { Slice 3 } & 1.1622-0.1810 \mathrm{i} & 1.0225-0.0344 \mathrm{i} \\ \text { Slice 4 } & 1.1983-0.2072 \mathrm{i} & 1.0315-0.0569 \mathrm{i} \\ \text { Slice 5 } & 1.2271-0.2277 \mathrm{i} & 1.0465-0.0637 \mathrm{i}\end{array}$

Table 3.1. Results for Dielectric constant of sea foam at frequencies of $10.7 \mathrm{GHz}$ and $37 \mathrm{GHz}$ for 5 2-D Slices of randomly packed airbubbles covered with thin-layer of seawater [2].

\section{FINITE DIFFERENCE ALGORITHM OF ALGORITHM OF PARABOLIC EQUATION}

The wide-angled and exponential pseudo-differential operator was approximated using a sum of Padé(1,1) functions as

$$
f(\sigma, x)=\exp (\sigma(\sqrt{1+x}))
$$

This is a function of the variable $x$, with complex parameter $\sigma$. Implementing a Taylor series expansion of the function $f$ in terms of the variable $x$, about the point $x=0$ yields 


$$
\begin{aligned}
f(\sigma, x) & =\exp (\sigma)\left\{1+\frac{\sigma}{2} x+\frac{\sigma(\sigma-1)}{4} \frac{x^{2}}{2 !}+\frac{1}{8}\left(3 \sigma-3 \sigma^{2}+\sigma^{3}\right) \frac{x^{3}}{3 !}\right. \\
& +\frac{1}{16}\left(-15 \sigma+15 \sigma^{2}-6 \sigma^{3}+\sigma^{4}\right) \frac{x^{4}}{4 !} \\
& \left.+0\left(x^{5}\right)\right\}
\end{aligned}
$$

where $\sigma=i k_{0} \Delta z$

$$
f_{1}(\sigma, x)=\exp (\sigma) \frac{1+a x}{1+b x}
$$

The Padé $(1,1)$ formula is obtained by matching the Taylor expressions to degree two (2) of $f$ and $f_{1}$. This is nicely done as shown

$$
\frac{1+a x}{1+b x}=1+\frac{\sigma}{2} x+\frac{\sigma(\sigma-1)}{4} \frac{x^{2}}{2 !}+0\left(x^{3}\right)
$$

The Padé $(1,1)$ coefficients $a$ and $b$ are

$$
a=\frac{1+\sigma}{4}, b=\frac{1-\sigma}{4}
$$

The approximation $f_{1}(\sigma, x)$ can be expressed in terms of Padé coefficients $a$ and $b$

$$
f_{1}(\sigma, x)=\exp \left(\sigma \left(\sqrt{1+x))}=\exp (\sigma) \frac{1+\frac{1+\sigma}{4} x}{1+\frac{1-\sigma}{4} x}\right.\right.
$$

The Split-step formal matching expression is as shown

$$
u(z+\Delta z, x)=\frac{1+\frac{1+\sigma}{4} x}{1+\frac{1-\sigma}{4} x} u(z, x)
$$

The implicit numerical integration scheme of (7) is given as

$$
\left\{1+\frac{1-\sigma}{4} x\right\} u^{n+1}=\left\{1+\frac{1+\sigma}{4} x\right\} u^{n}
$$

This was achieved by central finite differencing of the depth operator $Z_{h}$ with respect to the depth variable $z$. The depth variable is discretized as $z=z+(1 / 2) \Delta z$.

$$
\left(1+b Z_{h}\right) u^{n+1}=\left(1+a Z_{h}\right) u^{n}
$$

Further algebraic expression of (9) yields

$$
u^{n+1}+b Z_{h} u^{n+1}=u^{n}+a Z_{h} u^{n}
$$


Expression of $Z_{h} u^{n+1}$ and $Z_{h} u^{n}$ as sum of second-order partial differential equation and dielectric constant of the media is given below

$$
\begin{aligned}
& Z_{h} u^{n+1}=\frac{1}{k^{2}} \frac{\partial^{2} u^{n+1}}{\partial z^{2}}+\left(n^{2}-1\right) u^{n+1} \\
& Z_{h} u^{n}=\frac{1}{k^{2}} \frac{\partial^{2} u^{n}}{\partial z^{2}}+\left(n^{2}-1\right) u^{n}
\end{aligned}
$$

Applying second-order finite central difference method yields

$$
\frac{\partial^{2} u\left(z_{i}, x_{n}\right)}{\partial z^{2}}=\frac{-u_{i+2}^{n+1}+16 u_{i+1}^{n+1}-30 u_{i}^{n+1}+16 u_{i-1}^{n+1}-u_{i-2}^{n+1}}{12 \Delta z^{2}}
$$

Substituting the expression for $\frac{\partial^{2} u\left(z_{i}, x_{n}\right)}{\partial z^{2}}$ in (13) into (11) yields

$$
Z_{h} u^{n+1}=\frac{1}{12 k^{2} \Delta z^{2}}\left\{-u_{i+2}^{n+1}+16 u_{i+1}^{n+1}-30 u_{i}^{n+1}+16 u_{i-1}^{n+1}-u_{i-2}^{n+1}\right\}+\left(n^{2}-1\right) u_{i}^{n+1}
$$

Where $A=\frac{1}{12 k^{2} \Delta z^{2}}$ and $C=\left(n^{2}-1\right),(14)$ becomes

$$
Z_{h} u^{n+1}=A\left\{-u_{i+2}^{n+1}+16 u_{i+1}^{n+1}-30 u_{i}^{n+1}+16 u_{i-1}^{n+1}-u_{i-2}^{n+1}\right\}+C u_{i}^{n+1}
$$

and can be further expressed as

$$
Z_{h} u^{n+1}=-A u_{i+2}^{n+1}+16 A u_{i+1}^{n+1}-30 A u_{i}^{n+1}+C u_{i}^{n+1}+16 A u_{i-1}^{n+1}-A u_{i-2}^{n+1}
$$

Substituting $Z_{h} u^{n+1}$ in (16) into the left-hand side (LHS) of (10) gives

$$
u_{i}^{n+1}+b_{1}\left\{-A u_{i+2}^{n+1}+16 A u_{i+1}^{n+1}-30 A u_{i}^{n+1}+C u_{i}^{n+1}+16 A u_{i-1}^{n+1}-A u_{i-2}^{n+1}\right\}
$$

which is further expressed as 
$u_{i}^{n+1}+-b_{1} A u_{i+2}^{n+1}+16 b_{1} A u_{i+1}^{n+1}-30 A b_{1} u_{i}^{n+1}+b_{1} C u_{i}^{n+1}+16 b_{1} A u_{i-1}^{n+1}-b_{1} A u_{i-2}^{n+1}$

Taking like terms we obtain

$$
-b_{1} A u_{i+2}^{n+1}+16 b_{1} A u_{i+1}^{n+1}\left(-30 b_{1} A+b_{1} C+1\right) u_{i}^{n+1}+16 b_{1} A u_{i-1}^{n+1}-b_{1} A u_{i-2}^{n+1}
$$

Similarly, $Z_{h} u_{i}^{n}$ can be expressed as

$$
Z_{h} u_{i}^{n}=A\left\{-u_{i+2}^{n}+16 u_{i+1}^{n}-30 u_{i}^{n}+16 u_{i-1}^{n}-u_{i-2}^{n}\right\}+C u_{i}^{n}
$$

and

$$
u_{i}^{n}+Z_{h} u_{i}^{n}=u_{i}^{n}+a_{1}\left\{-A u_{i+2}^{n}+16 A u_{i+1}^{n}-30 A u_{i}^{n}+C u_{i}^{n}+16 A u_{i-1}^{n}-A u_{i-2}^{n}\right\}
$$

substituting equation (21) into the right-hand side (RHS) of (20) yields

$$
u_{i}^{n}-a_{1} A u_{i+2}^{n}+16 a_{1} A u_{i+1}^{n}-30 a_{1} A u_{i}^{n}+a_{1} C u_{i}^{n}+16 a_{1} A u_{i-1}^{n}-a_{1} A u_{i-2}^{n}
$$

Taking like terms gives

$$
-a_{1} A u_{i+2}^{n}+16 a_{1} A u_{i+1}^{n}+\left(-30 a_{1} A+a_{1} C+1\right) u_{i}^{n}+16 a_{1} A u_{i-1}^{n}-a_{1} A u_{i-2}^{n}
$$

Let's denote $\alpha=-a_{1} A, \beta=16 a_{1} A, \gamma=\left(-30 a_{1} A+a_{1} C+1\right), \zeta=-b_{1} A, \eta=16 b_{1} A$ and $\lambda=\left(-30 b_{1} A+b_{1} C+1\right)$

Therefore, equation (23) becomes

$$
\alpha u_{i+2}^{n+1}+\beta u_{i+1}^{n+1}+\gamma u_{i}^{n+1}+\beta u_{i-1}^{n+1}+\alpha u_{i-2}^{n+1}=\zeta u_{i+2}^{n}+\eta u_{i+1}^{n}+\lambda u_{i}^{n}+\eta u_{i-1}^{n}+\zeta u_{i-2}^{n}
$$

Equation (24) in matrix form is written as

$$
X_{m} U_{n+1}=Y_{m} U_{n}
$$


where $X_{m}$ and $Y_{m}$ are $m \times m$ pentadiagonal or tridiagonal matrices while $U_{n+1}$ and $U_{n}$ are column vectors of same dimension $m$. The animated form of (25) is shown below.

$$
\left(\begin{array}{ccccccc}
\gamma & \beta & \alpha & 0 & 0 & 0 & 0 \\
\beta & \gamma & \beta & \alpha & 0 & 0 & 0 \\
\alpha & \beta & \gamma & \beta & \alpha & 0 & 0 \\
0 & \alpha & \beta & \gamma & \beta & \alpha & 0 \\
0 & 0 & \alpha & \beta & \gamma & \beta & \alpha \\
0 & 0 & 0 & \alpha & \beta & \gamma & \beta \\
0 & 0 & 0 & 0 & \alpha & \beta & \gamma
\end{array}\right)\left(\begin{array}{c}
u_{i+1}^{n+1} \\
u_{i+1}^{n+1} \\
\cdot \\
\cdot \\
\cdot \\
\cdot \\
u_{i-N}^{n+1}
\end{array}\right)=\left(\begin{array}{ccccccc}
\lambda & \eta & \zeta & 0 & 0 & 0 & 0 \\
\eta & \lambda & \eta & \zeta & 0 & 0 & 0 \\
\zeta & \eta & \lambda & \eta & \zeta & 0 & 0 \\
0 & \zeta & \eta & \lambda & \eta & \zeta & 0 \\
0 & 0 & \zeta & \eta & \lambda & \eta & \zeta \\
0 & 0 & 0 & \zeta & \eta & \lambda & \eta \\
0 & 0 & 0 & 0 & \zeta & \eta & \lambda
\end{array}\right)\left(\begin{array}{c}
u_{i+1}^{n} \\
u_{i+1}^{n} \\
\cdot \\
\cdot \\
\cdot \\
\cdot \\
u_{i-N}^{n}
\end{array}\right)
$$

The solution to (26) is obtained by solving two pentadiagonal matrices. This can be solved using Gaussian elimination method, LU decomposition, inverse method, Jacobi's method, Gauss Siedel method, etc. Here, equation (26) was reduced by the matrix-vector product of $Y_{m}$ and $U_{n}$ to obtain $(A x=b)$ expressed in block matrix form as given below.

$$
(A)(x)=(b)
$$

The input parameters of algorithm comprise of $A(m \times m)$ non-singular square matrix and vector $b(m \times 1)$ on the RHS in the animation, the output solution vector is expressed as $x(m \times 1)$ returned at the end of the routine. The square pentadiagonal matrix $A$ have entries $\gamma, \beta$, and $\alpha$ while $b$ have $\lambda, \eta$, and $\zeta$. The parameters $\gamma, \beta$, and $\alpha$ were pre-computed using the expressions below.

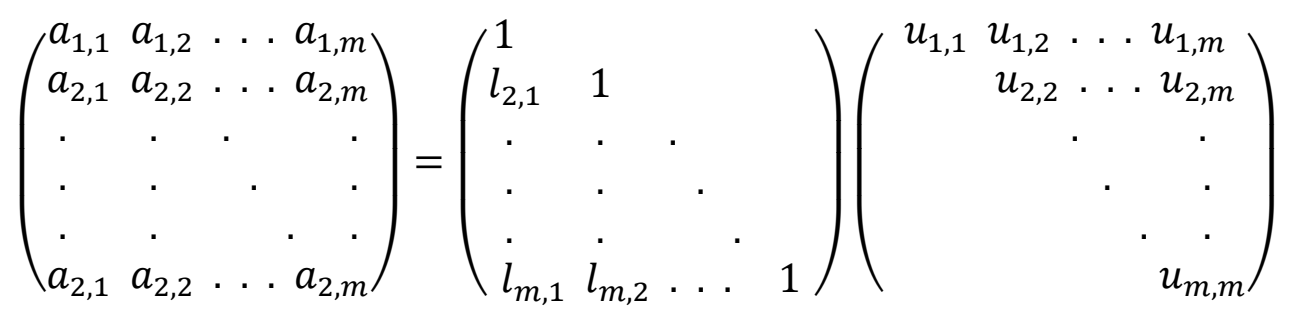




\section{Algorithm: Numerical Computation of Split-Step Padé $(N, M)$ Solution to EM Wave Propagation}

through Layers of Foam-Covered Sea-surface using LU Decomposition and Backward Substitution

Require: $A, b$

Ensure: $x$

1: procedure $\operatorname{LUMAT}(A, x, b)$

2: $\quad L U \leftarrow$ decomposition $(A)$ using Crout's algorithm

3: $\quad$ for $\boldsymbol{j}=1,2,3 \ldots . . N$ do

4: $\quad u_{1 j}=a_{1 j}$

5: end for

6: $\quad$ for $\boldsymbol{i}=1,2,3 \ldots . . N$ do

7: $\quad l_{i i}=1$

8: $\quad$ end for

9: $\quad$ for $\boldsymbol{i}=2,3 \ldots \ldots$ do

10: $\quad l_{i 1}=a_{i 1} / u_{11}$

11: end for

12: $\quad$ for $\boldsymbol{j}=1,2,3 \ldots . N$ do

13: $\quad$ for $\boldsymbol{i}=1,2,3 \ldots \ldots$ do

14:

$$
u_{i j}=a_{i j}-\sum_{k=1}^{i-1} l_{i j} u_{k j}
$$

15:

end for

16: $\quad$ for $i=j+1, j+2, \ldots N$ do

17:

$$
l_{i j}=\left(a_{i j}-\sum_{k=1}^{j-1} l_{i j} u_{k j}\right) / u_{j j}
$$

18:

$$
\text { end for }
$$

19: end for

20: $\quad$ for $\boldsymbol{i}=1: N$ do

21: $\quad x(:, i) \leftarrow \operatorname{LUBKSB}(\operatorname{LU}, \operatorname{IND} X, b(:, i)) \triangleright$ BackSubstitution

22: end for

23: end procedure 


\section{RESULTS AND FINDINGS}

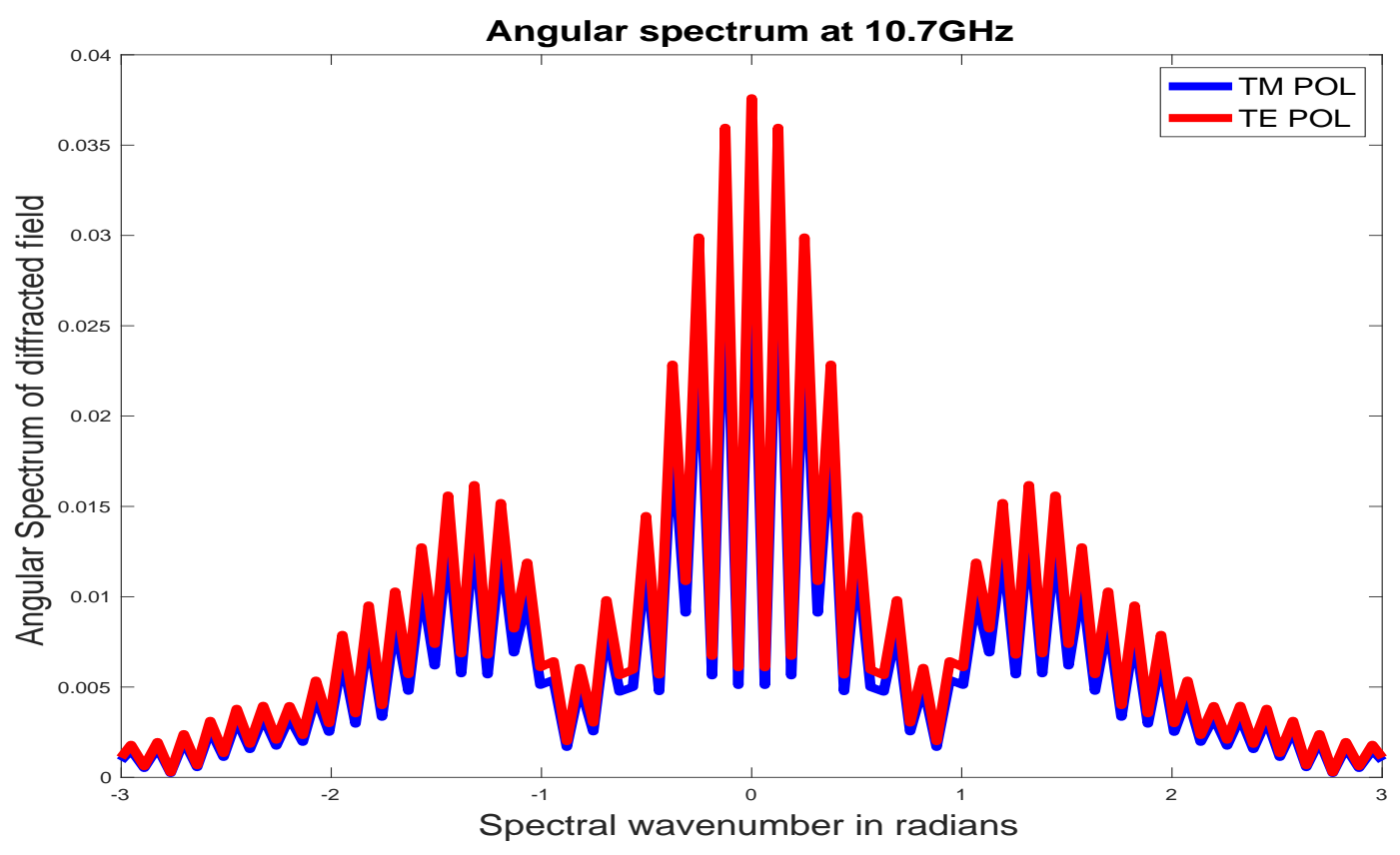

Figure 4.1: Angular Spectrum of Diffused Reflected Wave Field at $10.7 \mathrm{GHz}$.

In Figure 4.1, the angular spectrum of diffused reflected wave field as a function of spectral wavenumber in radians at $10.7 \mathrm{GHz}$ for both horizontal polarization (TE) and vertical polarization (TM) are shown. Here, the angular spectrum of the reflected wave fields at slice thickness $\delta_{t}=2 \mathrm{~mm}$ for a deep phase scattering screen is a function of the spectral wavelength $(p, q)$ for one dimensional $(1 D)$ problem. The broadening of the peaks in Figure 4.1 are attributed to $\exp \left(i \Delta z \sqrt{k^{2}-\left(p^{2}+q^{2}\right)}\right)$. When the $k^{2}$ approaches $p^{2}+q^{2}$, the peaks of the angular spectrum are spread or broadened across $(p, q)$ spectral domain. This happens as shown in Figure 4.1 at low WindSat frequency of $10.7 \mathrm{GHz}$ when $k^{2} \ll p^{2}+q^{2}$. However, it is obvious that the peaks of the diffused reflected wave field for (TE) mode are higher than that of (TM) mode. 


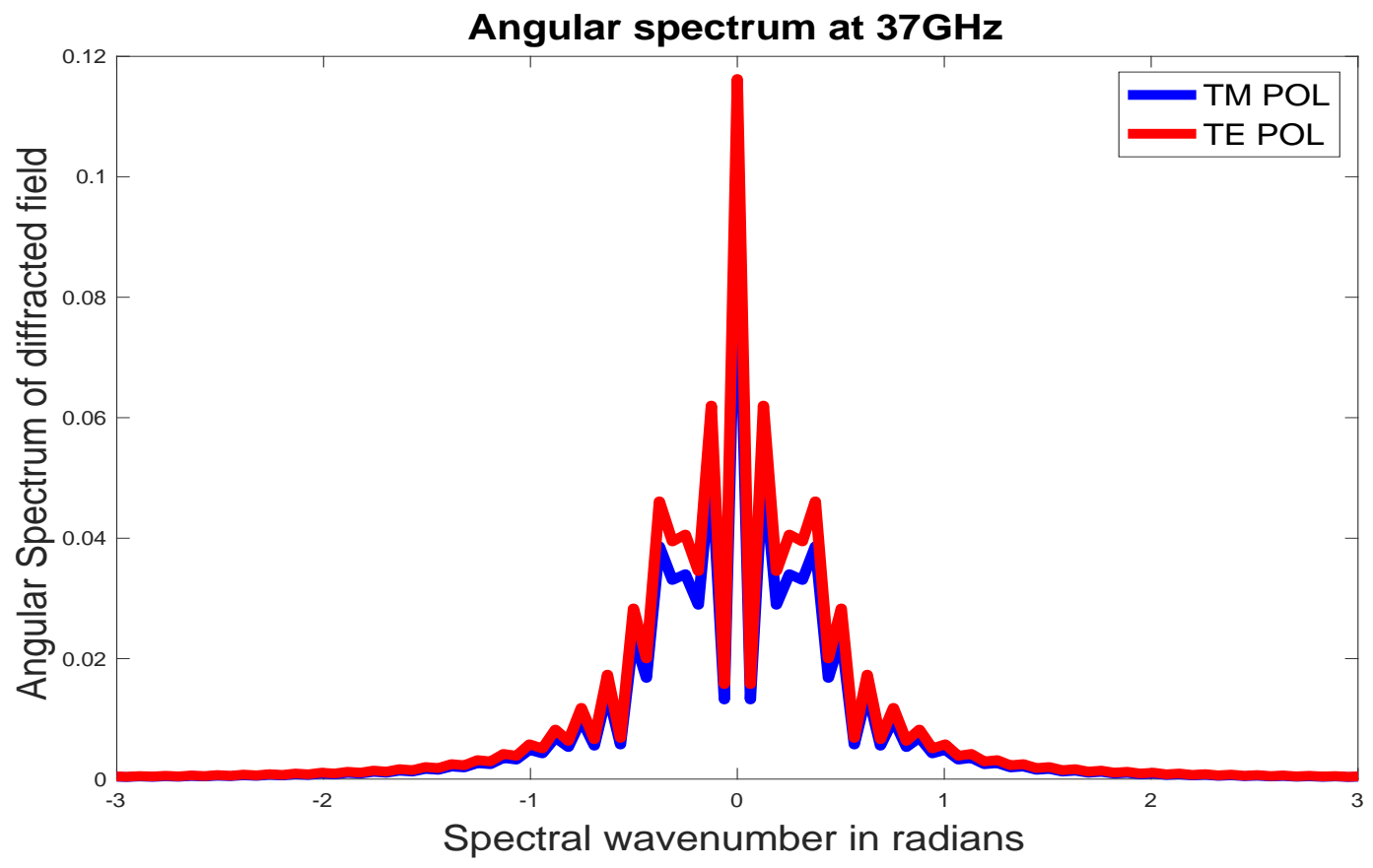

Figure 4.2: Angular Spectrum of Diffused Reflected Wave Field at $37 \mathrm{GHz}$.

The angular spectrum of diffracted wave field at $37 \mathrm{GHz}$ is shown in Figure 4.2. Figure 4.2 illustrate that the higher the frequency, the sharper and narrower the diffused reflected peaks because the diffraction effects of the scattering is reduced with increasing frequency. This is due to the relationship between the wavenumber $k$ and the spectral wavenumbers $\left(p^{2}+q^{2}\right)$. From the expression $k=2 \pi / \lambda$, where $\lambda$ is the wavelength of the incident wave field which decreases with increase in frequency. For propagation frequency $37 \mathrm{GHz}$, with effective dielectric constant of seafoam at various slices shown in Table 3.1, the wave field undergoes significant attenuation as it travels through slice 1 to slice 5. The amplitude of TE polarized wave field is higher than TM polarized wave field as shown in Figure 4.2. For thin phase scattering screen $\delta_{t}=0.2 \mathrm{~mm}$, the dominant factor for attenuation of the wave field is scattering at the sea surface. 


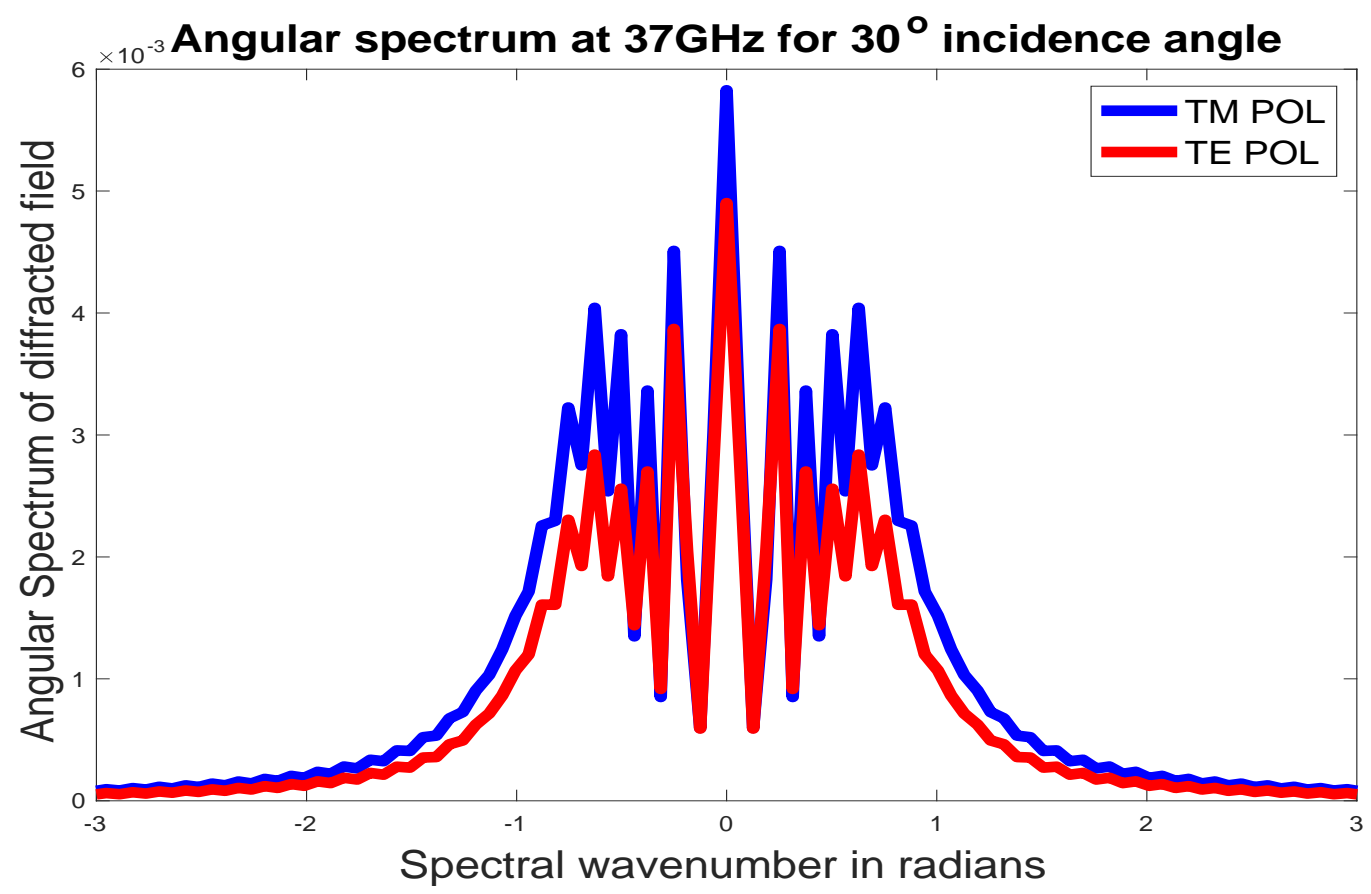

Figure 4.3: Angular Spectrum of Diffused Reflected Wave Field at $37 \mathrm{GHz}$ for Incidence Angle of $30^{\circ}$

In Figure 4.3, the angular spectrum of the scattered wave field at $37 \mathrm{GHz}$ for an incident angle of $30^{\circ}$ shows that for both TE and TM modes, there are more diffused reflections when compared to Figure 4.4 with an incident angle of $45^{\circ}$. It was observed that the attenuation of the field when it interacts with randomly distributed air-bubbles with different complex effective dielectric constants was due to scattering and increases with increase in the angle of incidence of the propagating wave field. Here, phase variations of the wave fields through foam layers with varying refractive indices appears incoherent which exemplifies diffuse scattering. Figure 4.4 shows that there are more interactions between the wave field and randomly distributed sea foams which can be attributed to scatterers which are closely packed with irregular boundaries and propagating angle of incident of $45^{\circ}$. 


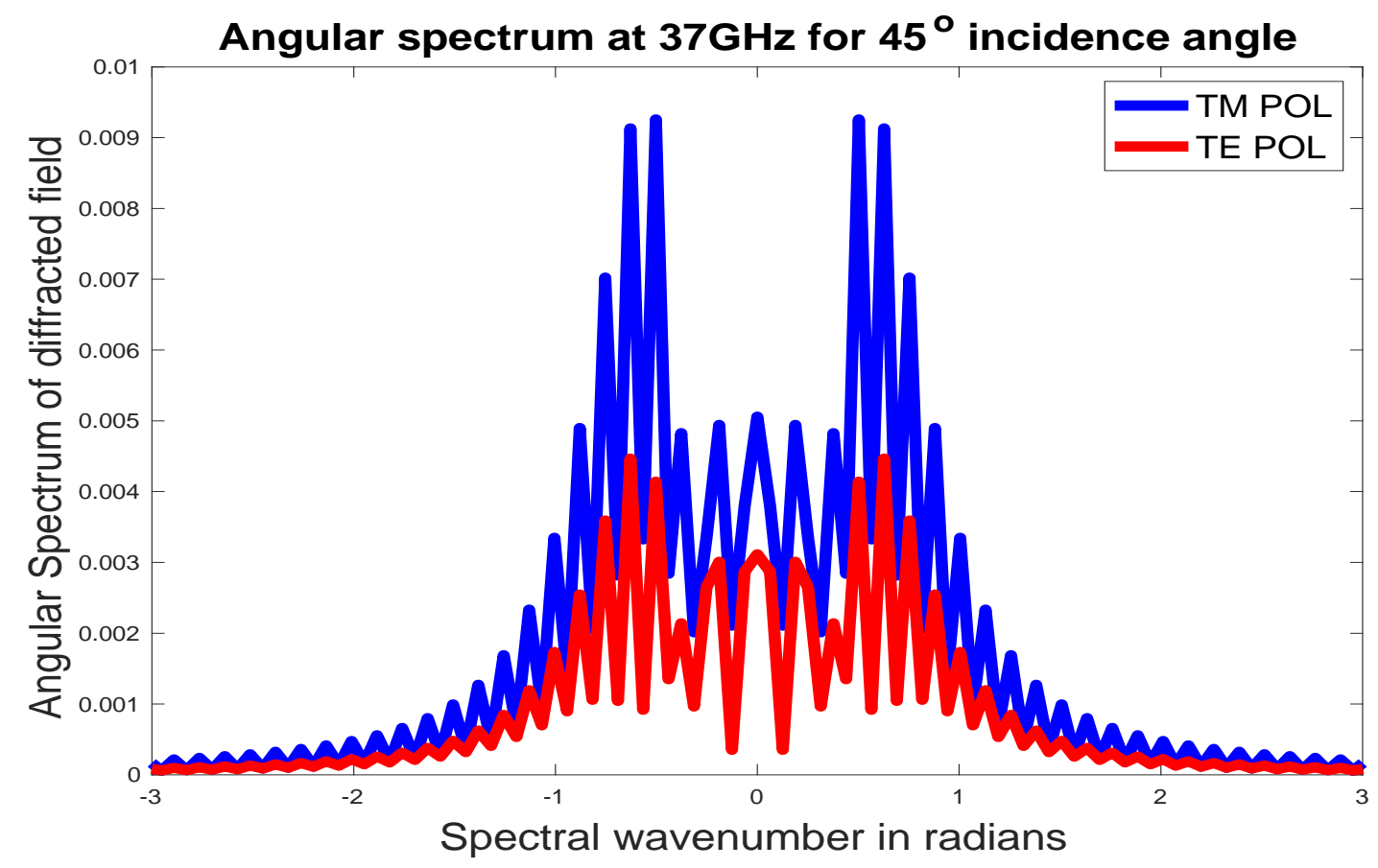

Figure 4.4: Angular Spectrum of Diffused Reflected Wave Field at $37 \mathrm{GHz}$ for Incidence Angle of $45^{\circ}$

\section{CONCLUSION}

We were able to illustrate that extinction of propagated wave field through thin phase scattering screens are due to diffused reflections by sea foam coved sea surface which builds up as the wave field travels through slices of sea foam layers. For thin phase scattering screens with low slice thickness $\left(\delta_{t}\right)$, scattering is the dominant factor for extinction of the wave field while absorption is responsible for extinction of the wave field for large slice thickness. Extinction of the wave field depend on frequency of the propagated field, slice thickness, depth of sea foam layer, effective dielectric constant of sea foam, foam void fraction, angle of incident wave field and polarization. 


\section{Acknowledgment}

\section{This research work was supported financially Dr. Nimibofa Ayawei. \\ References}

[1]. Benjamin, Ayibapreye Kelvin. "Evaluation of millimetre-wave coherent scattering from a sea surface covered by foam modelled as sequences of thin phase-scattering screens using split-step Fourier method." PhD diss., University of Essex, 2019.

[2]. Benjamin, Ayibapreye Kelvin, and Collins E. Ouserigha. "Extinction of Millimeter wave on Two Dimensional Slices of Foam-Covered Sea-surface." International Journal of Scientific Research and Development, vol. 3, no. 4, July-August -2020.

[3]. Tappert, F. D. "Parabolic equation method in underwater acoustics." The Journal of the Acoustical Society of America 55, no. S1 (1974): S34-S34.

[4]. Tappert, Frederick D, "Parabolic equation method modelling with the split-step Fourier transform in four dimensions" J. Acoust. Soc. Am 103, no. 2090 (1998): 4aUW6.

[5]. R. Hardin and F. Tappert, "Application of the split-step Fourier transform method to the numerical solution of nonlinear and variable coefficient wave equations," Siam Rev, vol. 15, no. 1, pp. 423-429, 1973.

[6]. Tolstoy, I. "Smoothed boundary conditions, coherent low-frequency scatter, and boundary modes." The Journal of the Acoustical Society of America 75, no. 1 (1984): $1-22$

[7]. Suarez, Pablo. "An introduction to the split step Fourier method using MATLAB." (2016).

[8]. Levy, Mireille. Parabolic equation methods for electromagnetic wave propagation. No. 45. IET, 2000.

[9]. Lee, Ding, George Botseas, and John S. Papadakis. "Finite-difference solution to the parabolic wave equation." The Journal of the Acoustical Society of America 70, no. 3 (1981): 795-80.

[10]. Collins, Michael D. "A split-step Padé solution for the parabolic equation method." The Journal of the Acoustical Society of America 93, no. 4 (1993): $1736-1742$.

[11]. Benjamin, A. K., and DH O. Bebbington. "Millimeter-wave propagation and attenuation in closed packed sea-foam layer and complex dielectric constant of seafoam using split-step Fourier transform." In 2017 Progress in Electromagnetics Research Symposium-Fall (PIERS-FALL), pp. 2556-2563. IEEE, 2017.

\section{Authors}

First Author - First Author- Ayibapreye Kelvin Benjamin, Ph. D in Computing and Electronic Systems University of Essex, United Kingdom, M.Sc. in Electronic Communications and Computer Engineering, The University of Nottingham, United Kingdom, B.Eng. Electrical/Electronic Engineering, Niger Delta University, Nigeria, Niger Delta University, Wilberforce Island Bayelsa State, Nigeria. ayibapreyebenjamin@ndu.edu.ng

Second Author-Collins E. Ouserigha, Ph. D in Condensed Matter Physics, University of Warwick, United Kingdom, M.Sc. in SolidState Electronics, University of Port Harcourt, Rivers State, Nigeria, B.Sc. Physics (Electronic Option), Niger Delta University, Nigeria, Niger Delta University, Wilberforce Island Bayelsa State, Nigeria. Ouserigha.ec@ndu.edu.ng

Third Author - Aguiyi Nduka Watson, M.Eng. in Electrical Engineering, Rivers State University, Nigeria, B.Eng. Electrical Engineering, Rivers State University, Nigeria, Federal University Otueke, Bayelsa State, Nigeria. aguiyiwatson@gmail.com

Correspondence Author - First Author- Ayibapreye Kelvin Benjamin, Ph. D in Computing and Electronic Systems University of Essex, United Kingdom, M.Sc. in Electronic Communications and Computer Engineering, The University of Nottingham, United Kingdom, B.Eng. Electrical/Electronic Engineering, Niger Delta University, Nigeria, Niger Delta University, Wilberforce Island Bayelsa State, Nigeria. ayibapreyebenjamin@ndu.edu.ng, contact number: +2348166924395. 